\title{
Resource-Aware Multimedia Content Delivery: A Gambling Approach
}

\author{
Mouna Allani ${ }^{1}$, Benoît Garbinato ${ }^{1}$ and Fernando Pedone ${ }^{2, *}$ \\ ${ }^{1}$ Information Systems Institute (ISI), University of Lausanne, University of Lausanne (UNIL), CH-1015 \\ Lausanne, Switzerland \\ ${ }^{2}$ Faculty of Informatics, University of Lugano, University of Lugano (USI), CH-6900 Lugano, Switzerland \\ *Corresponding author: fernando.pedone@unisi.ch
}

\begin{abstract}
In this paper, we propose a resource-aware solution to achieving reliable and scalable stream diffusion in a probabilistic model, i.e. where communication links and processes are subject to message losses and crashes, respectively. Our solution is resource-aware in the sense that it limits the memory consumption, by strictly scoping the knowledge each process has about the system, and the bandwidth available to each process, by assigning a fixed quota of messages to each process. We describe our approach as gambling in the sense that it consists in accepting to give up on a few processes sometimes, in the hope of better serving all processes most of the time. That is, our solution deliberately takes the risk not to reach some processes in some executions, in order to reach every process in most executions. The underlying stream diffusion algorithm is based on a tree-construction technique that dynamically distributes the load of forwarding stream packets among processes, based on their respective available bandwidths. Simulations show that this approach pays off when compared to traditional gossiping, when the latter faces identical bandwidth constraints.
\end{abstract}

Keywords: large-scale systems, reliable streaming, resource awareness

Received 10 October 2008; revised 27 February 2009

Handling editor: Franco Zambonelli

\section{INTRODUCTION}

Reliable stream diffusion under constrained environment conditions is a fundamental problem in large-scale multimedia content delivery. In this context, the efficiency of a given content delivery solution directly depends on the performance of its underlying multicast protocol. Environment conditions are typically constrained by the reliability and the capacity, usually limited, of its components. Nodes and communication links can fail, unexpectedly ceasing their operation and dropping messages, respectively. Moreover, real-world deployment does not offer nodes and links infinite memory and infinite bandwidth. Therefore, realistic solutions should use local storage and inter-node communication sparingly, and account for node crashes and message losses.

In this paper, we investigate the problem of reliable stream diffusion in unreliable and constrained environments from a novel angle. Our approach is probabilistic: with high probability, all consumers will be reached and all information addressed to them delivered; however, there is no guarantee that this will happen. Different from previous probabilistic algorithms found in the literature, we resort to a 'gambling approach,' which deliberately penalizes a few consumers in rare cases, in order to benefit most consumers in common cases. We show experimentally that the approach pays off in that it outperforms traditional gossip-based algorithms (GBA) when subject to similar environment constraints.

The key idea of our solution is to stream multimedia content according to a global propagation graph. This graph approximates a global tree aiming at the maximum reachability and efficient use of the available bandwidth. The approach is completely decentralized: nodes build propagation trees, which we call maximum probability trees (MPTs), autonomously. Several MPTs are dynamically composed to achieve a global graph reaching most (hopefully all) consumer nodes. This solution is scalable and based on a composition of local optimums, i.e. each MPT ensures the maximum probability of reaching all processes in its subgraph when subject to bandwidth constraints. MPTs are composed in a manner that respects bandwidth constraints, and the MPT construction is fully parameterized. Nodes are free to define the scope of their local knowledge, from direct neighborhood to the entire network. The scope of each process can be defined according 
to its local constraints (e.g. processing power, memory capacity).

Besides discussing a new reliable stream diffusion algorithm, we also show that it can be implemented in a very modular way, lending itself to real deployment. Our solution consists in decomposing the problem of reliable stream diffusion into sub-problems. This separation of concerns gives rise to an architecture composed of five layers.

The remainder of this paper is organized as follows. In Section 2 we introduce the system model and define the problem that motivates this work. Section 3 describes our reliable streaming solution based on a tree-construction technique. Section 4 describes a performance evaluation of our approach, including an analysis of the costs and benefits of gambling. We discuss related work in Section 5. Finally, in Section 6 we summarize our findings and conclude with some final remarks.

\section{SCALABLE RESOURCE-AWARE STREAMING}

Stream diffusion is a typical 3-step scenario: (1) the producer breaks the outgoing stream into elemental messages (stream packets) and multicasts them to interested consumers, (2) intermediate nodes route these messages to the consumers and (3) each consumer recomposes the received messages into a coherent incoming stream. This is depicted in Fig. 1. In a resource-constrained environment, the main challenge then consists in routing stream messages in a way that efficiently uses available resources.

\subsection{Basic system model}

We consider an asynchronous distributed system composed of processes (nodes) that communicate by message passing. Our model is probabilistic in the sense that processes can crash and links can lose messages with a certain probability. More formally, we model the system's topology as a connected graph $G=(\Pi, \Lambda)$, where $\Pi=\left\{p_{1}, p_{2}, \ldots, p_{n}\right\}$ is a set of processes of size $n$ and $\Lambda=\left\{l_{1}, l_{2}, \ldots\right\} \subseteq \Pi \times \Pi$ is a set of bidirectional communication links. Process crash probabilities and message loss probabilities are modeled as failure configuration $C=$ $\left(P_{1}, P_{2}, \ldots, P_{n}, L_{1}, L_{2}, \ldots, L_{|\Lambda|}\right)$, where $P_{i}$ is the probability that process $p_{i}$ crashes during one computation step and $L_{j}$ is the probability that link $l_{j}$ loses a message during one communication step.

\subsection{Problem statement}

Intuitively, the main question addressed in this paper is the following: how can we make stream messages reach all consumers with a high probability, in spite of unreliable processes and links, and of the limited resources (e.g. bandwidth) available to each process?

Formally, the limited resources constraint is modeled as $Q=\left(q_{1}, q_{2}, \ldots, q_{n}\right)$, the set of quotas associated to processes in the system. Each individual quota of messages $q_{i}$ represents the number of messages process $p_{i}$ is able to send in order to forward a single stream packet. A quota may represent a set of physical constraints related to the limited hardware resources or a dedicated percentage of these resources fixed by the peer itself. This percentage captures the fact that the user behind a peer can voluntarily limit the resources dedicated to the P2P streaming service. In other words, a quota is a translation of both the percentage of hardware resources a peer is willing to dedicate to forward a stream packet and the upload limit of the ISP of the peer, which might be further limited by the percentage of that bandwidth the peer is willing to dedicate to the streaming

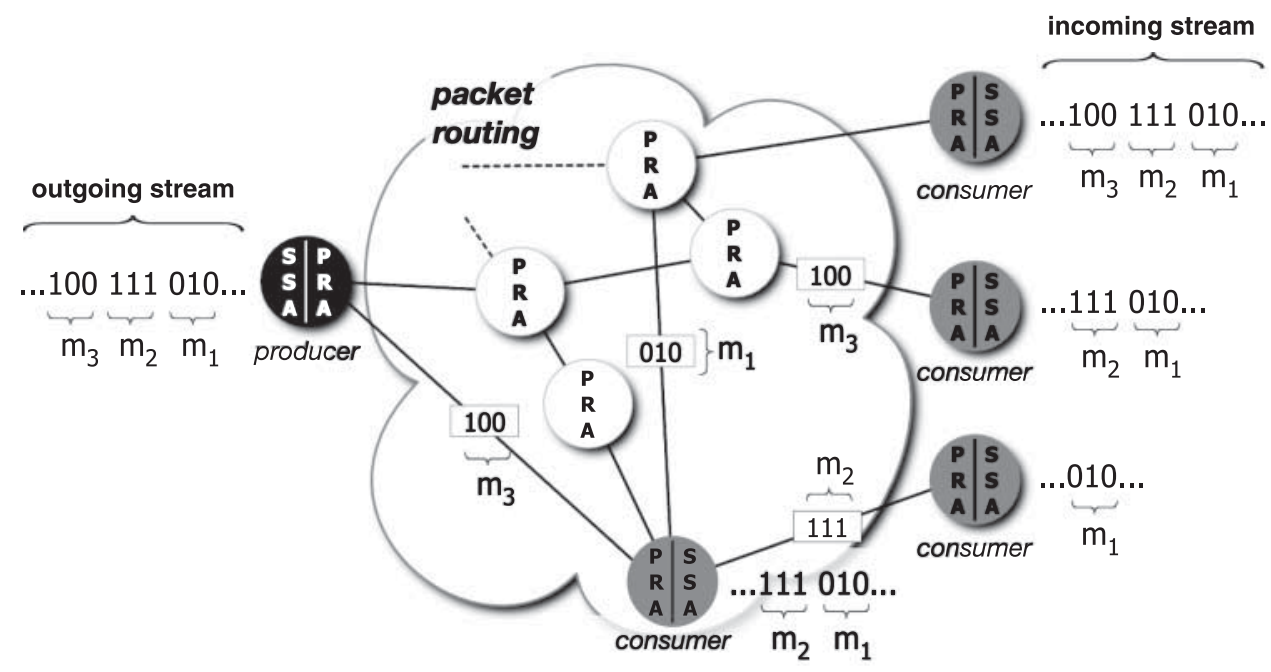

FIGURE 1. Multimedia content delivery—stream diffusion scenario. 
service. By extending the basic system model presented earlier, we then can say that the tuple $S=(\Pi, \Lambda, C, Q)$ completely defines the system considered in this paper.

In order to take into account processing and memory constraints, we further assume that each process has only a partial view of the system, meaning that its routing decisions can only be based on incomplete knowledge. Formally, the limited knowledge of process $p_{i}$ is modeled with distance $d_{i}$, which defines the maximum number of links in the shortest path separating $p_{i}$ from any other node in its known subgraph. Distance $d_{i}$ implicitly defines the partial knowledge of $p_{i}$ as scope $s_{i}=\left(\Pi_{i}, \Lambda_{i}, C_{i}, Q_{i}\right)$, with $\Pi_{i} \subseteq \Pi, \Lambda_{i} \subseteq \Lambda$, $C_{i} \subseteq C$ and $Q_{i} \subseteq Q$. In the remainder of this paper, any graph comprised of processes and links should be understood as also including the corresponding configuration and quota information.

Based on the above definitions, we can now restate the problem we address in this paper more succinctly: given its limited scope $s_{i}$, how should process $p_{i}$ use its quota $q_{i}$ in order to contribute to reach all consumers with a high probability?

\section{A GAMBLING APPROACH}

In the absence of any constraints on resources, making stream messages reach all processes with a high probability is quite easy, typically via some generous gossiping (or even flooding) algorithm. In a large-scale resource-constrained system, however, such a solution is not realistic.

\subsection{Diffusion trees as starting point}

The starting point of our approach can be found in [1], where we proposed an algorithm to efficiently diffuse messages in a probabilistically unreliable environment. Intuitively, the solution consists in building a spanning tree that contains the most reliable paths connecting all processes, using a modified version of Prim's algorithm [2]. The algorithm is also somehow resource-aware in that it tries to minimize the number of messages necessary to reach all processes with a given probability.

This algorithm, however, does not limit the bandwidth: when asking the algorithm to diffuse a message with a high probability in a very unreliable environment, the number of messages tends to explode. Furthermore, this solution does not limit memory consumption either: in order to achieve optimality, it requires a complete knowledge of the system topology and of the failure probabilities associated to links and processes. Informally, the approach presented hereafter consists in building a diffusion graph that exhibits properties similar to that of [1], while taking into account strict constraints on resources (bandwidth, memory etc). As presented in Section 2, these constraints are modeled via $q_{i}$ and $s_{i}$, respectively, the limited quota and the limited scope available at each process $p_{i}$.
As soon as we face resource constraints, we have to make difficult decisions. In the context of this paper, this observation translates into deciding how high the risk is which we are willing to take, in order to increase our chances to reach all consumers. More specifically, the question we ask ourselves is the following: does it pay off to take the risk to sacrifice a few consumer processes in some executions, in order to reach every process in most executions? As we shall see in Section 4, when comparing the performance of our solution to that of a typical gossiping approach, the answer is clearly yes.

Intuitively, our approach consists in having processes make bold decisions, in spite of their limited view of the system (scope), in the hope to better use the available resources (quota). That is, along the paths from the producer to the consumers, a process $p_{i}$ may decide to build a local propagation tree based on its limited scope $s_{i}$ in order to maximize the probability of reaching everybody in $s_{i} .{ }^{1}$ In building its local propagation tree, $p_{i}$ also decides how processes in $s_{i}$ should use their quotas. Since these decisions can be made concurrently, process $p_{i}$ has no guarantee that processes in $s_{i}$ will actually follow its decisions. As we shall see in Section 4, this approach can lead to some (fairly rare) executions in which some processes are never reached. Experiments show however that the benefits of taking such a risk pays off in most executions.

\subsection{Solution overview}

Our solution is based on the five-layer architecture pictured in Fig. 2. The top layer represents a standard stream fragmentation layer. It executes the scalable streaming algorithm (SSA) and is responsible for breaking the outgoing stream into a sequence of messages on the producer side and for assembling these messages back into an incoming stream on the consumer side. Roughly speaking, this layer corresponds to the Transport layer in the OSI model [3]. The SSA layer then relies on the packet routing algorithm (PRA), which is responsible for routing stream messages through a propagation graph covering the whole system; this layer corresponds to the Network layer in the OSI model. This propagation graph results from the spontaneous aggregation of various propagation trees concurrently computed by some intermediate routing processes defined as responsible for this task. As suggested by Fig. 2, producers and consumers execute both the SSA and PRA layers, while pure routing processes only execute the PRA layer. The responsibility for building propagation trees is delegated to the propagation tree algorithm (PTA), which in turn relies on the partial view delivered by the environment modeling layer $(E M L)$. The latter relies on Bayesian inference to approximate the environment within distance $d_{i}$ of each process $p_{i}$. Explaining how the environment modeling actually

\footnotetext{
${ }^{1}$ The actual criteria that determines whether $p_{i}$ will make such a decision or not is explained later.
} 


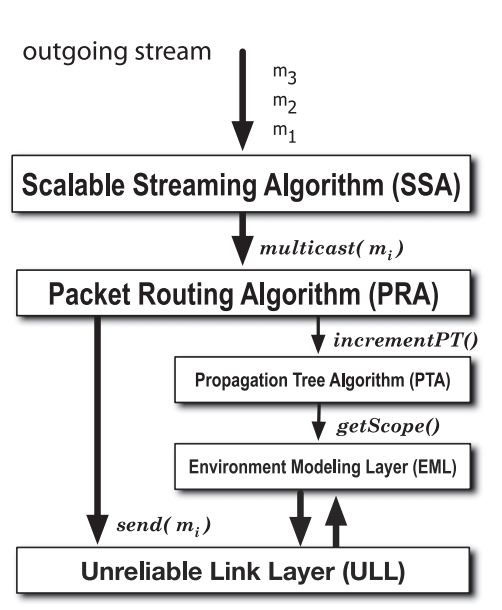

(a)

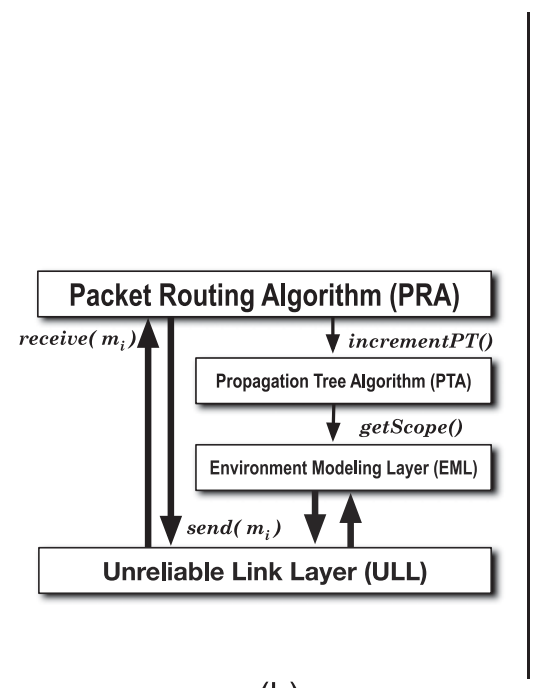

(b)

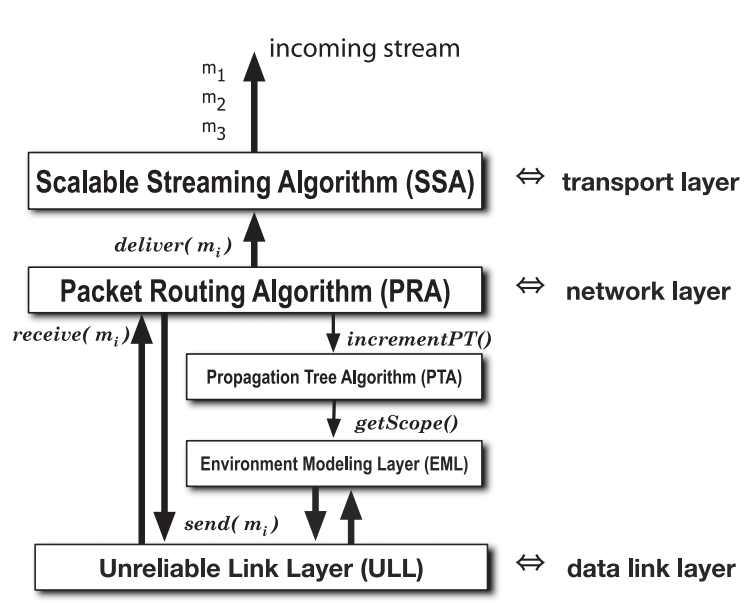

(c)

FIGURE 2. A layered architecture: (a) producer and router, (b) pure router and (c) consumer and router.

works falls beyond the scope of this paper and can be found in [1]. Finally, the unreliable link layer (ULL) allows each process $p_{i}$ to send messages to its direct neighbors in a probabilistically unreliable manner. This layer corresponds to the Data Link layer of the OSI model.

\subsection{Scalable streaming algorithm}

The scalable streaming solution, presented in Algorithm 1, is fairly straightforward. On the producer side, as long as some data is available from the outgoing stream (line 6), the algorithm reads that data, builds up a message containing it and multicasts the message using the multicast() primitive of the PRA layer (lines 7-10). On the consumer side, upon receiving a message from PRA (line 11), the algorithm writes the data contained in that message to the incoming stream, provided that the message is not out of sequence (lines 12-14). Because of the probabilistic

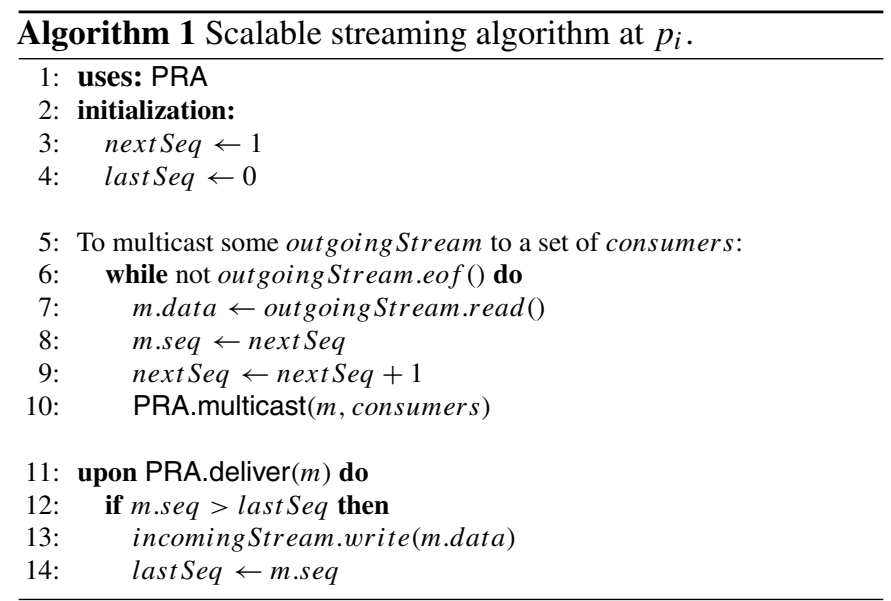

nature of our environment, messages can indeed be received out of sequence, in which case they are simply dropped. This is the standard way to handle out-of-sequence packets when streaming real-time data, such as audio or video streams. Note that this strategy can be easily improved by a simple local buffering mechanism in order to deal with jitter and out-of-order messages.

\subsection{Packet routing algorithm}

The packet routing solution, presented in Algorithm 2, CONSISTS in disseminating stream messages through a propagation graph generated in a fully decentralized manner. This propagation graph actually results from the spontaneous aggregation of several propagation trees. Each propagation tree is in turn the result of an incremental building process carried out along the paths from the producer to the consumers. It is important to note however that the aggregated propagation graph itself might well not be a tree.

\subsubsection{On the producer}

The routing process starts with producer $p_{i}$ calling the multicast () primitive (line 4). As a first step, $p_{i}$ asks the PTA layer to build a first propagation tree $p t$, using the increment $P T$ () primitive (line 5). This primitive is responsible for incrementing the propagation tree passed as argument, using the scope of the process executing it (here $p_{i}$ ). Since $p_{i}$ is the producer, the initial propagation tree passed as argument is simply composed of $p_{i}$ and its associated information (failure probability $P_{i}$ and quota $q_{i}$ ). As discussed in Section 3.5, the returned propagation tree $p t$ maximizes the probability of reaching everybody in scope $s_{i}$, based on available quotas. Process $p_{i}$ then calls the optimize() primitive, passing it $p t$ 


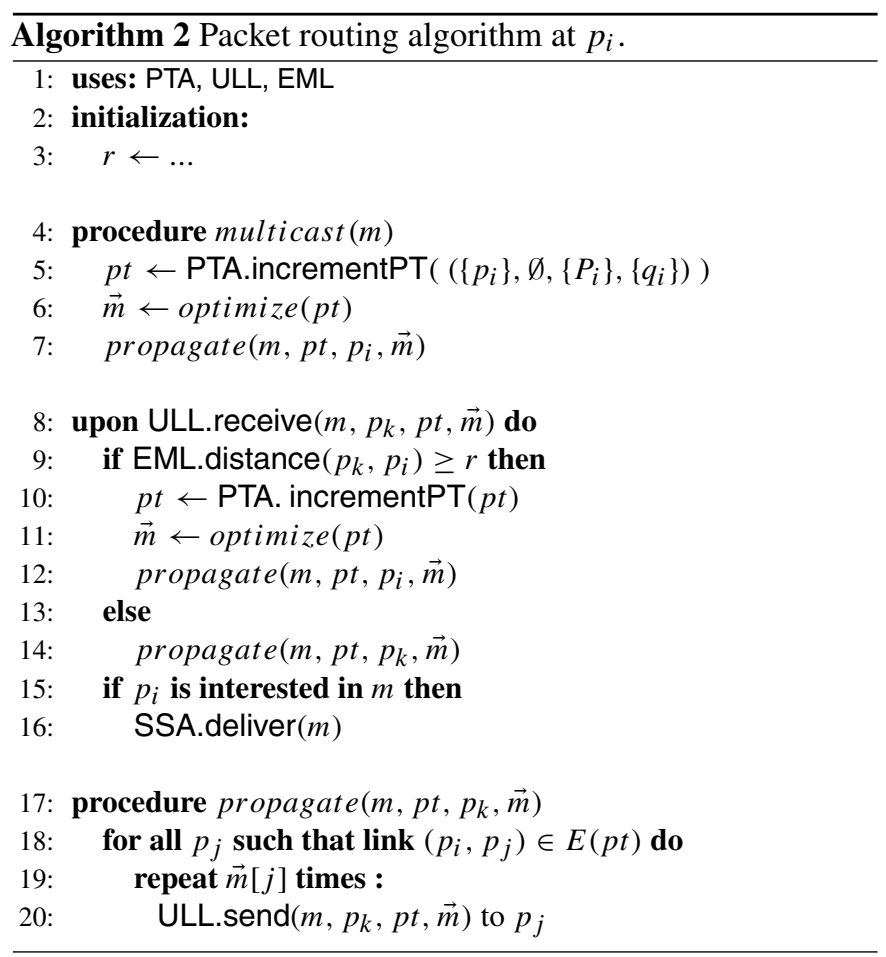

(line 6). This primitive is discussed in detail in Section 3.7. At this point, all we need to know is that it returns a propagation vector $\vec{m}$ indicating, for each link in $p t$, the number of messages that should be sent through that link in order to maximize the probability of reaching everybody in scope $s_{i}$. Finally, $p_{i}$ calls the propagate () primitive (line 7 ), which simply follows the forwarding instructions computed by optimize(). That is, it sends stream message $m$, together with some additional information, to $p_{i}$ 's children in $p t$. As we shall see below, this additional information is used throughout the routing process to build up the propagation graph.

\subsubsection{On the consumer}

When a consumer $p_{i}$ receives message $m$, together with the aforementioned information (line 8), it has to first decide whether to increment $p t$ before further propagating $m$ (lines 10-12), or to simply follow the propagation tree $p t$ it just received (line 14). The propagation tree $p t$ should be incremented if and only if the distance that separates $p_{i}$ from $p_{k}$, the process that last incremented $p t$, is equal to $r \leq d_{k}$, the increment rate. In such a case, $p_{i}$ is said to be an incrementing node.

Intuitively, $r$ defines how often a propagation tree should be incremented as it travels through the propagation graph. The latter then spontaneously results from the concurrent and uncoordinated increments of propagation trees finding their way to the consumers. Finally, process $p_{i}$ delivers message $m$ to the SSA layer only if it is interested in it (lines 15 and 16). If this is not the case, process $p_{i}$ is merely a router node.

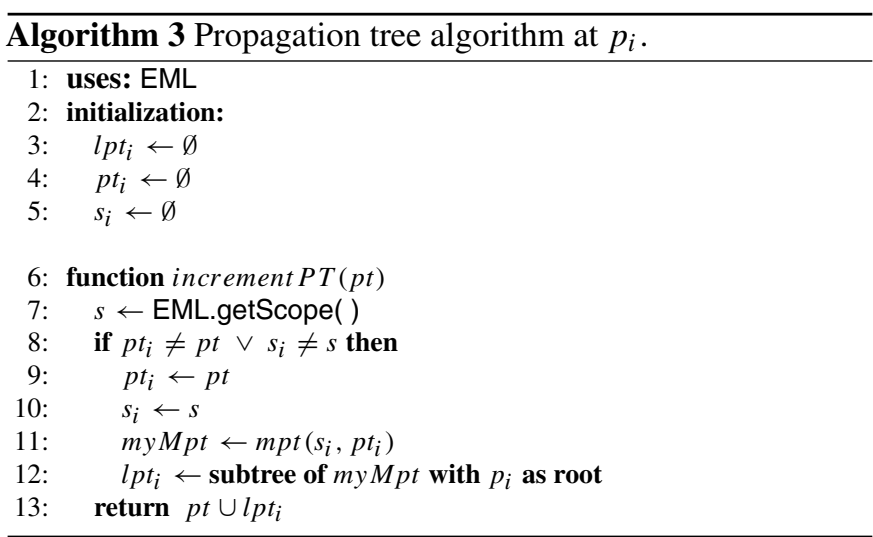

\subsection{Propagation tree algorithm}

The solution to increment propagation trees is encapsulated in the incrementPT() primitive, presented in Algorithm 3. This primitive takes a propagation tree $p t$ as argument and increments it if needed, i.e. if something changed in the environment of $p_{i}$ or if $p t$ is different from the propagation tree that was last incremented (line 8). The conditional nature of this increment is motivated by performance and resource concerns: during stable periods of the system, propagation trees remain unchanged, cutting down the processing load of incrementing nodes. To get an up-to-date view of its surrounding environment, $p_{i}$ calls the getScope () primitive provided by EML (line 7).

To build the local tree $l p t_{i}$, process $p_{i}$ first builds a $M P T$, using the $m p t()$ primitive (line 11). Details about the notion of MPT, and primitive $m p t$ (), are provided in Section 3.7. Briefly, MPT maximizes the probability of reaching every process within a given scope, by taking into account not only the intrinsic reliability of processes and links in scope $s_{i}$, but also the individual quotas available to processes in $s_{i}$. Note that primitive $m p t$ () increments $p t$ as a whole (see discussion below), whereas Algorithm 3 is in fact only interested in the subtree rooted at $p_{i}$ (line 12). This subtree is precisely the local tree $l p t_{i}$.

\subsection{The gambling effect}

Intuitively, the approach taken by the $m p t$ () primitive consists in augmenting $p t$ with the best branches in scope $s_{i}$, even if some of these branches are not downstream from $p_{i}$. These latter branches are said to be concurrent branches. This approach somehow consists in taking the risk to exclude some consumers from the propagation graph by accident. Process $p_{i}$ has indeed no way to inform processes located along concurrent branches about its incremental decisions, and has no guarantee that incremental decisions will be taken coherently with respect to each other. In order to partially mitigate this risk, Algorithm 3 merges the local tree with the original propagation tree passed as argument (line 13), rather than directly returning the maximum reliability tree (MRT). 


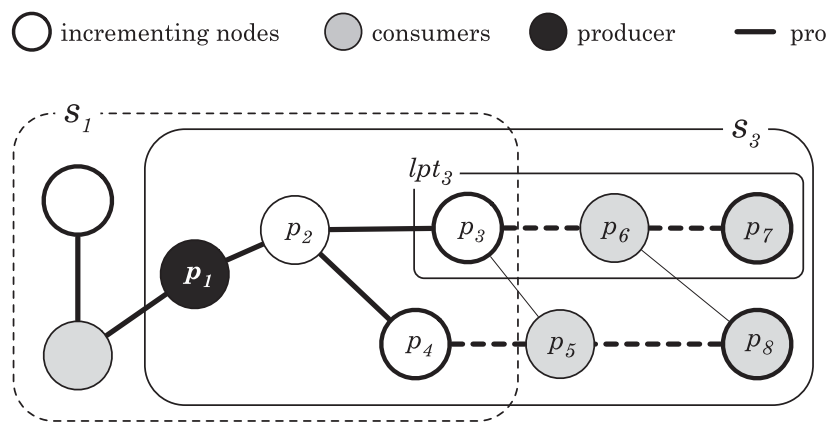

(a)

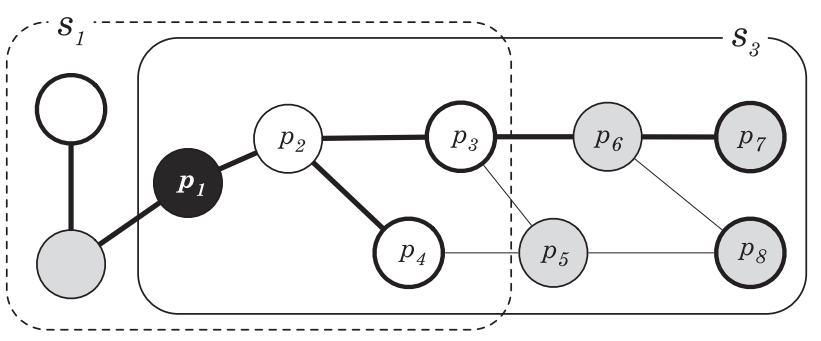

(b)

FIGURE 3. Propagation tree increment: (a) before the return statement and (b) returned propagation tree.

\subsubsection{Execution example}

Figure 3 illustrates the incrementing of the propagation tree on a simple example. In this scenario, the distance defining the scope and the increment rate $r$ are the same for all processes and equal to 2 . Process $p_{1}$, the producer, builds a first propagation tree $p t_{1}$ covering its scope $s_{1}$; this tree is pictured in Fig. 3a using bold links. All nodes in $p t_{1}$ that are at a distance $r=2$ from $p_{1}$ are incrementing nodes, which means they have to increment $p t_{1}$ when they receive it. Process $p_{3}$ being such a node, it calls the $m p t$ () function, passing it $p t_{1}$ and its scope $s_{3}$. This function adds the dashed links pictured in Fig. 3a to $p t_{1}$ and returns the resulting MPT; this MPT contains the local propagation tree rooted at $p_{3}$, i.e. $l p t_{3}$. The latter is then extracted from the MPT, merged with the initial propagation tree $p t_{1}$ and returned. Figure $3 b$ pictures the new propagation tree resulting from the above increment process.

\subsection{Maximum probability tree}

The concept of MPT is at the heart of our approach, as it materializes the risk taken during the construction of the propagation graph. Intuitively, an MPT maximizes the probability of reaching all processes within a given scope by optimally using the quotas of these processes. Before describing how the mpt () function given in Algorithm 4 builds up an MPT, we first recall the notions of reachability probability and reachability function.

\subsubsection{Reachability probability}

The reachability function, denoted $R()$, computes the probability of reaching all processes in some propagation tree $T$ with configuration $C(T)$, given a vector $\vec{m}$ defining the number of messages that should transit through each link of $T$. We then define the probability returned by $R()$ as $T$ 's reachability probability. Equation (1) proposes a simplified version of the reachability function borrowed from [1] - this version assumes that only links can fail by losing messages

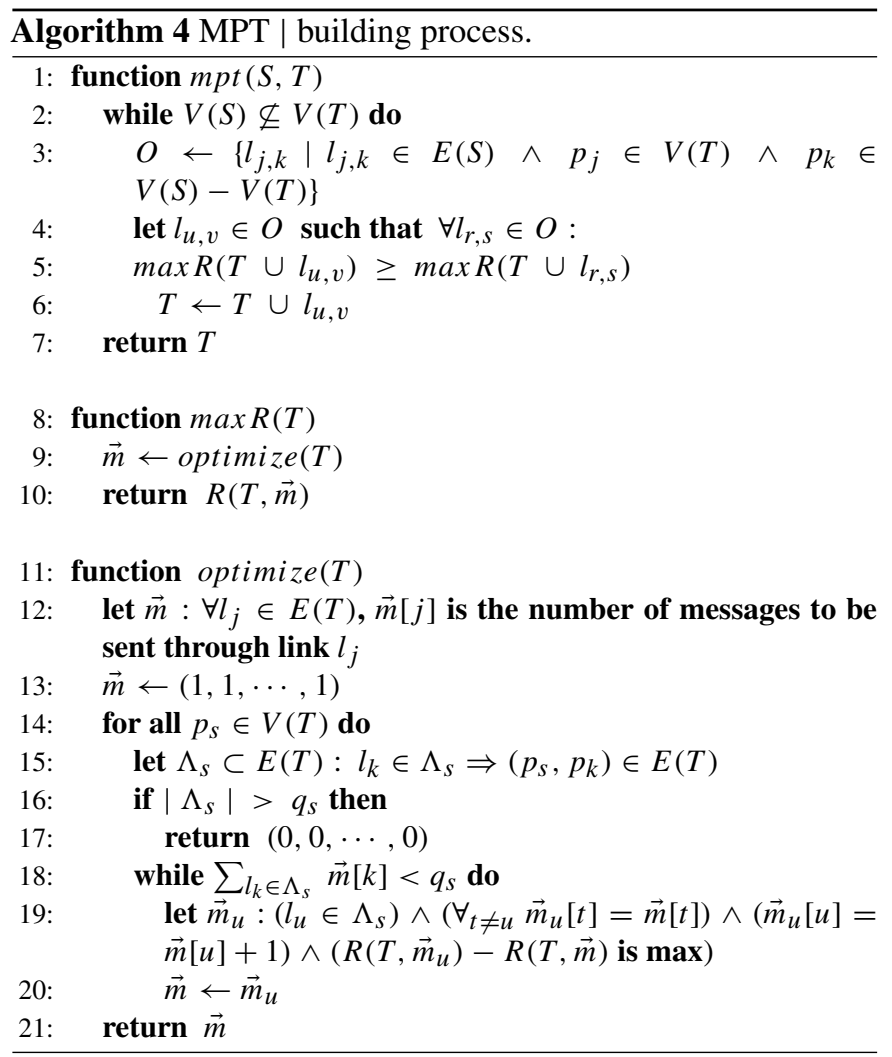

with a given probability, whereas processes are assumed to be reliable. $^{2}$

$$
R(T, \vec{m})=\prod_{j=1}^{|\vec{m}|} 1-L_{j}^{m[j]}, \quad \text { where } L_{j} \in C(T) .
$$

Using $R()$, we then define the $\max R()$ function presented in Algorithm 4 (lines 8-10), which returns the maximum reachability probability for $T$. To achieve this, $\max R()$ first calls the optimize() function in order to obtain a vector $\vec{m}$

\footnotetext{
${ }^{2}$ Note that this simplification causes no loss of generality; see [1] for details.
} 
that optimally uses the quotas available to processes in $T$. It then passes this vector, together with $T$, to $R()$ and returns the corresponding reachability probability.

The optimize() function iterates through each process $p_{s}$ in $T$ and divides individual quota $q_{s}$ in a way that maximizes the probability of reaching direct children of $p_{s}$ (lines 1420). For this, the function optimize() allots messages of $q_{s}$ one by one, until all messages have been allocated (lines 1820). That is, in each iteration step it chooses the outgoing link $l_{u}$ from $p_{s}$ that maximizes the gain in the probability of reaching all $p_{s}$ 's children in $T$, when sending one more message through $l_{u}$ (line 19). When all individual quotas have been allocated, optimize() returns a vector $\vec{m}$ that provides the maximum reachability probability when associated with $T$.

\subsubsection{MPT building process}

We now have all the elements needed to present the MPT building process carried out by $m p t($ ), given a scope $S$ and an initial propagation tree $T$. This function simply iterates until all processes in $S$ but not in $T$ have been linked to $T$, i.e. it only stops when $T$ covers the whole scope $S$ (lines 2-6). In each iteration step, the $\operatorname{mpt}()$ function then adds the link that produces a new tree exhibiting the maximum reachability probability (line 5).

\subsubsection{Execution example.}

Figures 4-6 illustrate the MPT building process based on a simple example. In this example, the initial tree $T$ is composed of only process $p_{1}$ and $S$ is the scope of $p_{1}$, i.e. $S=s_{1}$. During

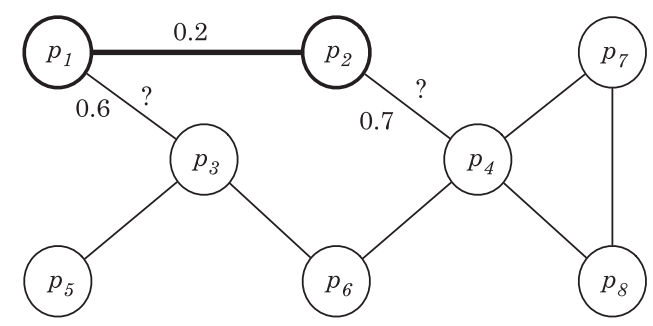

FIGURE 4. Resulting tree after the first iteration step.

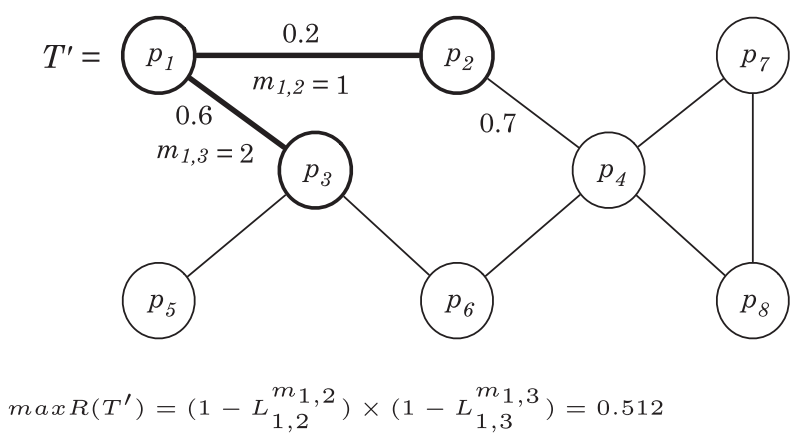

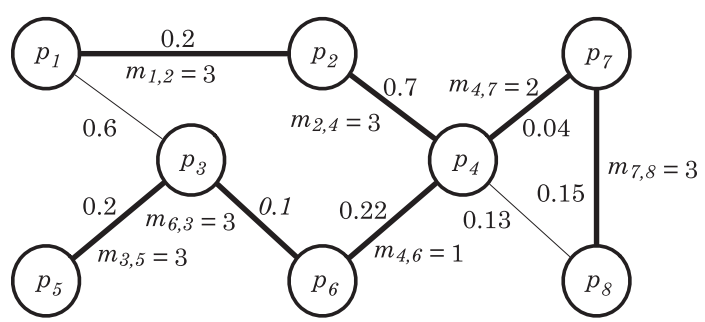

FIGURE 6. Final MPT.

the first iteration step, the algorithm simply chooses the most reliable link, i.e. link $l_{1,2}$ with failure probability $L_{1,2}=0.2$. At this point, it means that the entirety of $p_{1}$ 's quota has been allocated to reach $p_{2}$. In this example, the quota is identical for all processes and equal to 3 , i.e. $\forall p_{i}: q_{i}=3$.

At the beginning of the second step, the algorithm faces two alternatives: either adding the link $l_{1,3}$ and splitting the quota of $p_{1}$ between links $l_{1,2}$ and $l_{1,3}$, or adding the link $l_{2,4}$ and using the entirety of $q_{2}$, the quota of $p_{2}$, to reach $p_{4}$. These two alternatives are pictured in Fig. 5 as trees $T^{\prime}$ and $T^{\prime \prime}$, respectively.

Based on the result of the function $\max R()$, the algorithm chooses to keep $T^{\prime \prime}$, since it is the tree that offers the maximum probability of reaching everybody. Note however that this decision implies adding the link $l_{2,4}$ rather than the link $l_{1,3}$, although the latter is more reliable. Figure 6 pictures the final MPT returned by function $m p t()$.

\section{PERFORMANCE EVALUATION}

The performance of our scalable algorithm was evaluated through a simulation model. For simplicity, we only considered link failures, while assuming that processes are reliable, i.e. $\forall p_{i}: P_{i}=0$. As mentioned in Section 3.7, this does not compromise the generality of our approach. We performed experiments with processes organized in various topologies: we started from a ring where each process had two neighbors and then incrementally augmented the number of neighbors until reaching a connectivity of 20 neighbors per process. This provided a spectrum of possibilities for the evaluations, starting

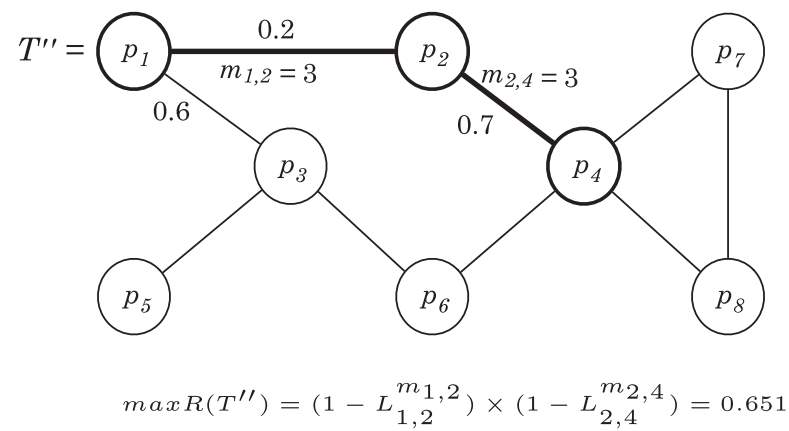

FIGURE 5. Alternative trees during the second iteration step. 
with a worst-case topology with respect to process distances (i.e. the ring), and gradually reducing the mean distance between processes in the system by adding more links. Unless mentioned otherwise, we assumed topologies with 100 processes.

To facilitate the evaluation, we set the scope to be the same for all processes during the execution, i.e. $\forall p_{i}: d_{i}=d$. To avoid regular network configurations, we then defined $20 \%$ of processes to be hubs. A hub has twice the quota of a normal process and is connected to its neighbors through highly reliable links, i.e. we set the message loss probability of these links to $10^{-4}$. Our performance evaluation consists in measuring the success rate of 1000 distinct executions. We consider an execution to be a success when the multicasted stream packet reaches all nodes in the system, i.e. the success rate is precisely what the notion of reachability probability tries to capture.

\subsection{Benefits of gambling}

Multicast protocols fall into two categories, those based on structured information dissemination such as our SSA, and those based on unstructured information dissemination, typically the case of gossip-based protocols. To measure the benefit of our gambling approach, we compare the SSA with a typical GBA, modified to implement the notion of individual quota: to propagate an incoming message $m$, the algorithm repeats the following two steps until exhausting its quota: (1) randomly choose a neighbor among those that did not yet acknowledge $m$ and (2) send $m$ to those neighbors. For the comparison, we then set the quota to 5 and the failure probability of each link ${ }^{3}$ to a random value within [0.05, 0.55]. As for specific parameters of SSA, we set the scope to 5 and the increment rate to 2 .

Figure 7 shows the evolution of the success rate of SSA and GBA, respectively, when varying the network connectivity. As we can see, the success rate of GBA decreases as the connectivity increases. This is due to the fact that each process randomly uses its quota of messages, without taking into account the reliability of links. Indeed, as the connectivity increases, it becomes more and more important to maximize the impact of each message on the overall reachability probability.

In contrast, for SSA, the success rate tends to increase with the network connectivity because SSA has a larger choice of links when computing local MPTs, and thus more chances to build a global propagation graph with a favorable reachability probability. Furthermore, even if some processes have a number of neighbors that exceeds their quota, our approach still tries to maximize the overall reachability probability by adapting the number of children of each process to its quota. As shown in Fig. 7, this has a significant impact on the actual success rate. For a connectivity of 20 for example, which is four times higher than the quota used in our experiments, the success rate is close to 100. In this figure, however, we can also see a drop in the

\footnotetext{
${ }^{3}$ To be more precise: each link that is not attached to a hub.
}

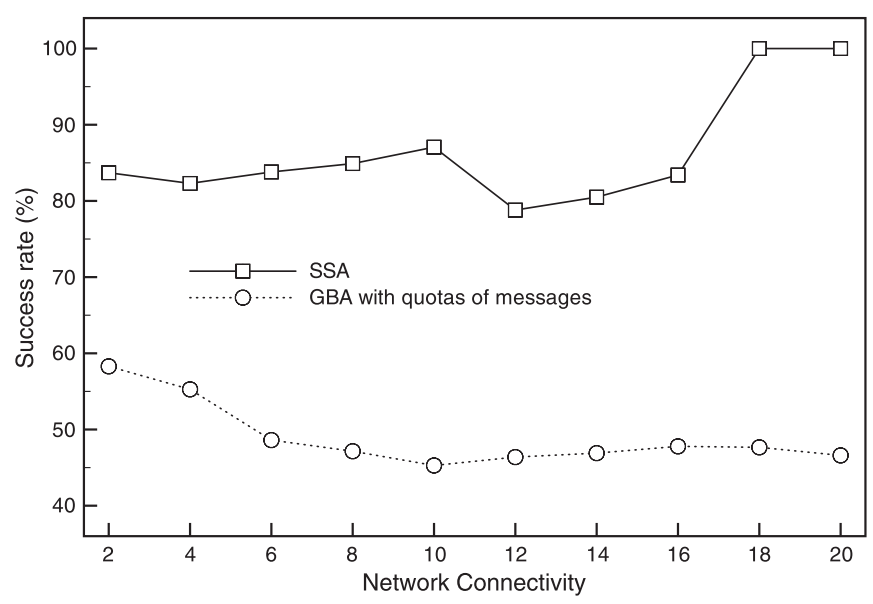

FIGURE 7. SSA vs. GBA with quotas-success rate.

success rate for connectivities between 10 and 16. As discussed hereafter, this drop constitutes the costs of gambling.

\subsection{Cost of gambling}

To evaluate the cost of our gambling approach, we introduce the notion of a missed execution of our algorithm. Such an execution, also simply called a miss, is one where at least one node in the system never received the multicast packet. We can further categorize such misses as either probabilistic misses or gambling misses. Probabilistic misses are caused by unreliable links sometimes losing messages, i.e. they are due to the probabilistic nature of the model we consider. Gambling misses on the other hand happen when the effective propagation graph does not cover the whole system. An effective propagation graph results from the aggregation of effectively followed propagation trees.

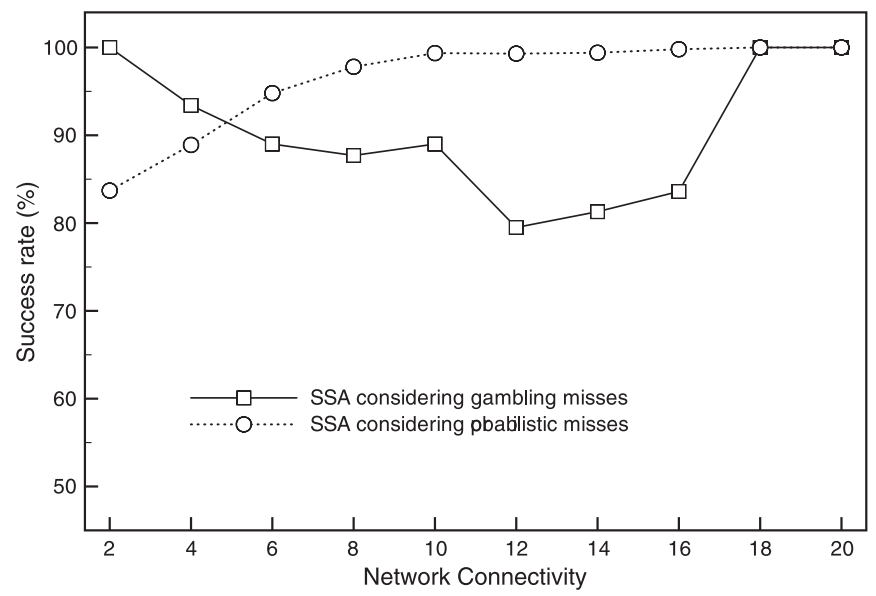

FIGURE 8. Influence of probabilistic misses and gambling misses on SSA's success rate. 


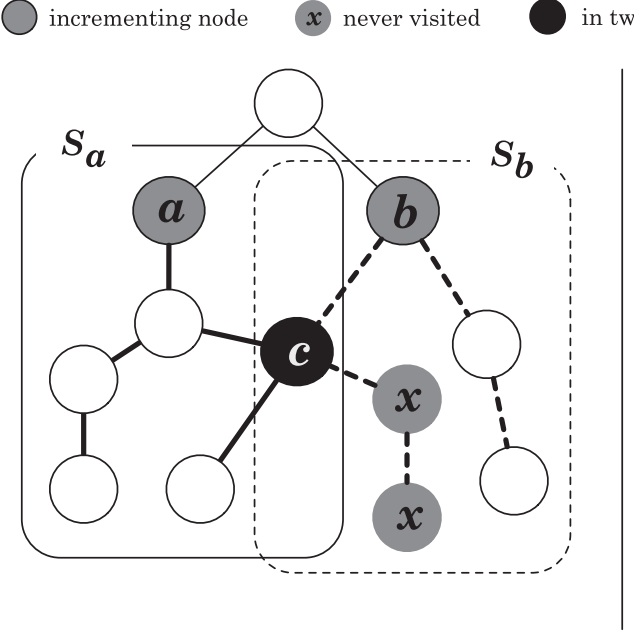

(a)

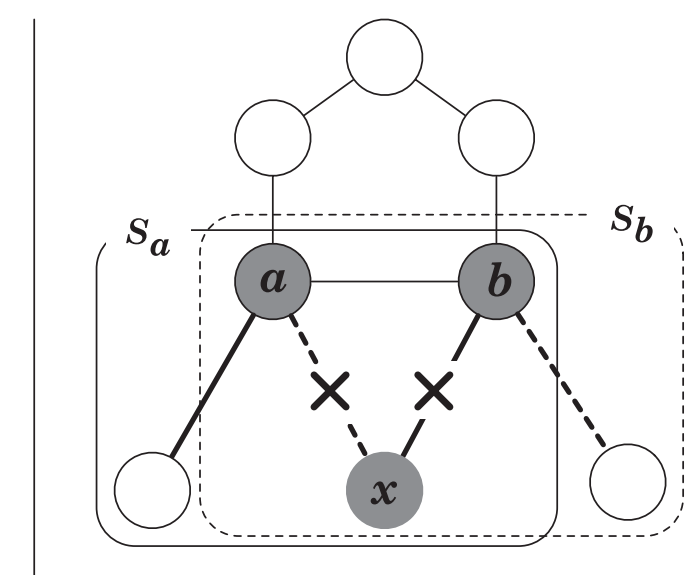

(b)

FIGURE 9. Conflicts causing gambling misses: (a) cyclic conflict (detected) and (b) mutual delegation conflict (not detected).

In Fig. 8, we show how probabilistic misses and gambling misses influence the success rate of our algorithm, i.e. the two curves presented in this figure result from the decomposition of the SSA curve presented in Fig. 7. Considering probabilistic misses, we can observe that as the connectivity increases, the probability of reaching all nodes also increases. This is not surprising, because as the connectivity increases, the number of links increases and the algorithm has a larger choice of links when computing $M P T$ and thus more chances to get an $M P T$ with a favorable reachability probability. In contrast, for gambling misses, as the connectivity increases, misses due to the structure of the effective propagation graph become more frequent because the algorithm has a larger choice of links, which induces a higher risk of making contradictory decisions when building distinct propagation trees. However, when reaching a high connectivity (12 links or more in our example), gambling misses become less frequent because the scope of each process becomes close to the whole system. ${ }^{4}$

Gambling cost mitigation The good news is that many cases of gambling misses are detectable and can be mitigated via a simple countermeasure, which leads to a few nodes exceeding their quotas. As discussed in Section 2.2, we assume that a quota of a node is not defined as the whole node propagation capacity. It can represent either a part of its capacity or the percentage of resources the peer allocated to the streaming service. As we have just seen, a gambling miss occurs when the resulting effective propagation graph does not cover the whole system. Such misses can

\footnotetext{
${ }^{4}$ When the scope covers the whole system, the propagation graph corresponds to the MPT built by the producer and covering the whole system. In this case there is no gambling involved.
}

be caused by two types of conflicting situations, pictured in Fig. 9.

A cyclic conflict, illustrated in Fig. 9a, is caused by the inclusion of some node $c$ into two distinct propagation trees. When $c$ receives a propagation tree, it uses its quota to propagate the packet in that tree, not knowing that a second tree will reach it. Thus, when the second propagation tree reaches $c$, the absence of the remaining quota can cause some descendants of $c$ in the second tree to never be reached. In Fig. 9a, node $c$ receives two conflicting propagation trees, first one computed by node $a$ and another computed by node $b$. As a consequence, nodes below $c$ in the tree computed by $b$ might never be reached. It is easy to see that upon reception of the second tree, $c$ is able to detect the conflict and to apply the countermeasure described hereafter.

A mutual delegation conflict, illustrated in Fig. 9b, is caused by contradictory decisions about how to include a given node, when incrementing two distinct propagation trees. In Fig. 9b, node $a$ decides to delegate the task of reaching node $x$ to node $b$, while $b$ decides to delegate the task of reaching $x$ to $a$. As a consequence, node $x$ will never be reached. As incrementing nodes do not inform each other about their respective incrementing decisions, the mutual delegation conflict is not detected.

\subsubsection{Cyclic conflict countermeasure}

As already suggested, we can mitigate cyclic conflicts by occasionally having some nodes exceed their quotas. It is interesting to note, however, that the detection of a cyclic conflict by some node $c$ does necessarily imply that some nodes might not be reached. More precisely, there exists two independent cases that require node $c$ to exceed its quota. The first case is straightforward and occurs when a descendant node of $c$ in the second propagation tree is not in the first propagation tree. This case is formalized by Condition 2. The second case, 


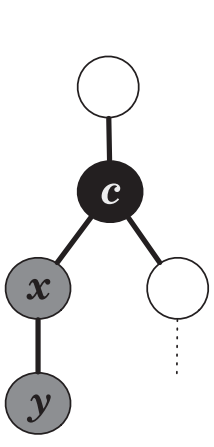

(a)

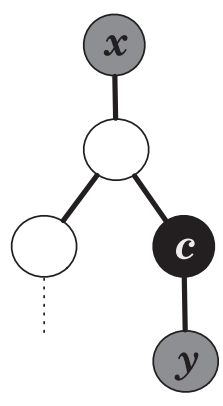

(b)
FIGURE 10. Two conflicting propagation trees received by $c$ : (a) propagation tree $T_{1}$ (received first) and (b) propagation tree $T_{2}$ (received later).

formalized by Condition 3, is more complex and explained by the example of Fig. 10.

$$
\begin{aligned}
& \exists y \in T_{2} \text {.children }(c)-T_{1} \\
& \left.\exists y \in\left(T_{2} \text {.children }(c) \cap T_{1}\right)\right) \\
& \quad \wedge\left(x \in T_{2} \mid c \in T_{2} \cdot \operatorname{children}(x) \wedge\left(y \in T_{1} \cdot \operatorname{children}(x)\right)\right)
\end{aligned}
$$

In Fig. 10, node $c$ first receives tree $T_{1}$ and later $T_{2}$. When receiving $T_{2}$ from node $x, c$ detects that node $y$ might not be reached. Indeed, in both trees $T_{1}$ and $T_{2}, y$ is a descendant of $c$. However, $x$ is a descendant of $c$ in $T_{1}$ and an ancestor of $c$ in $T_{2}$. Therefore, when $c$ receives $T_{2}$ from $x$, it deduces that $x$ was either not reached in $T_{1}$ or reached but decided to re-transmit through $T_{2}$. In both cases, $c$ should re-transmit, and hence exceed it quota, in order to reach $y$ or in order to reach the node for which $x$ decided to re-transmit.

\subsubsection{Countermeasure evaluation}

When evaluating the effectiveness of our solution to mitigate gambling costs, we compared the final success rate of experiments implementing the proposed countermeasure with experiments that do not. In doing so, we varied the network connectivity $c$, while fixing the incrementing rate $r$ to 2, the scope $d$ defining the known subgraph of each process to 5 , the range of loss probability $L_{i}$ to $[0.05,0.55]$ and the quota of messages $q_{i}$ to 5 .

Fig. 11a shows the success rate of executions implementing our countermeasure by varying the network connectivity, while Fig. $11 \mathrm{~b}$ shows the corresponding average number of exceeded quotas based on 1000 distinct executions. When comparing the curves of Fig. 11a and Fig. 8 that show the success rate considering the gambling misses, ${ }^{5}$ we can see that our countermeasure significantly improves the final result. Furthermore, as shown in Fig. 11b, the average number of exceeded quotas is negligible, i.e. less than one for 1000 distinct executions.

\footnotetext{
${ }^{5}$ Executions corresponding to these two curves have the same parameters.
}

\subsection{Benefits of combined adaptiveness}

This section discusses the advantage of combining both resource and unreliability awareness when building the propagation tree, that is, it shows the benefit of our MPT-construction technique. We compare our MPT with two relevant solutions. The first solution is inspired by the tree defined in Overcast [4]. Overcast is targeted at bandwidth-intensive applications. It defines a tree overlay that aims to maximize the bandwidth by placing nodes as far as possible from the root (the source) without sacrificing bandwidth. The available bandwidth resource in Overcast is modeled as weights assigned to links. In order to adapt the Overcast tree-construction technique to our model, we consider the link weight as the number of messages assigned to the link, calculated by dividing the node quota by the number of its outgoing links in the tree. Thus, when building the Overcast tree in our model, at each iteration we add the link through which we can assign the maximum number of messages.

The second protocol is part of our previous work defined in [1] defining a reliable broadcast taking into account nodes' failure probabilities $\left(P_{i}\right)$ and links' message loss probabilities $\left(L_{i}\right)$. This broadcast solution is also based on a tree overlay named the MRT. This tree defines the most reliable tree of a known subgraph through which a message will be propagated. To avoid compromising this protocol, we assume for this comparison that each node will be able to send at least one message to each of its children in a tree. Thus, as shown in Fig. 12a, each node's quota of messages is equal to the number of direct neighbors, i.e. $\forall q_{i}, q_{i}=c$, the network connectivity.

When it comes to the limited knowledge each node has about the system, we assume that, in both our strategy and the compared protocols, nodes have only a partial view. Based on this knowledge, we use our gambling increment strategy in order to build a propagation graph covering the whole system while using the different tree building criteria. Then, we apply our countermeasure to mitigate the gambling misses and focus our comparison in probabilistic misses. For a fair comparison, we also apply our Optimize() function both to the Overcast tree and the MRT. Thus, once our comparison trees are built, all the nodes' quotas are distributed in a way to maximize the advantage of this resource.

In this comparison, we vary the network connectivity $c$, while fixing the incrementing rate $r$ to 2, the scope $d$ defining the known subgraph of each process to 5 and the range of loss probability $L_{i}$ to $[0.05,0.55]$. As shown in Fig. 12, the success rate of our approach is higher when using MPT than when using the Overcast tree and the MRT. When the quota is equal to the network connectivity $c$ (Fig. 12a), the success rate of our approach and its comparison protocols increases as the $c$ increases and thus as $q_{i}$ increases. This reflects the capacity of available resources to hide the environment unreliability. When the quota $q_{i}$ is fixed to five messages (Fig. 12b), our approach provides a higher reliability when using $M P T$ than when using the Overcast tree. In addition, our approach has a different 


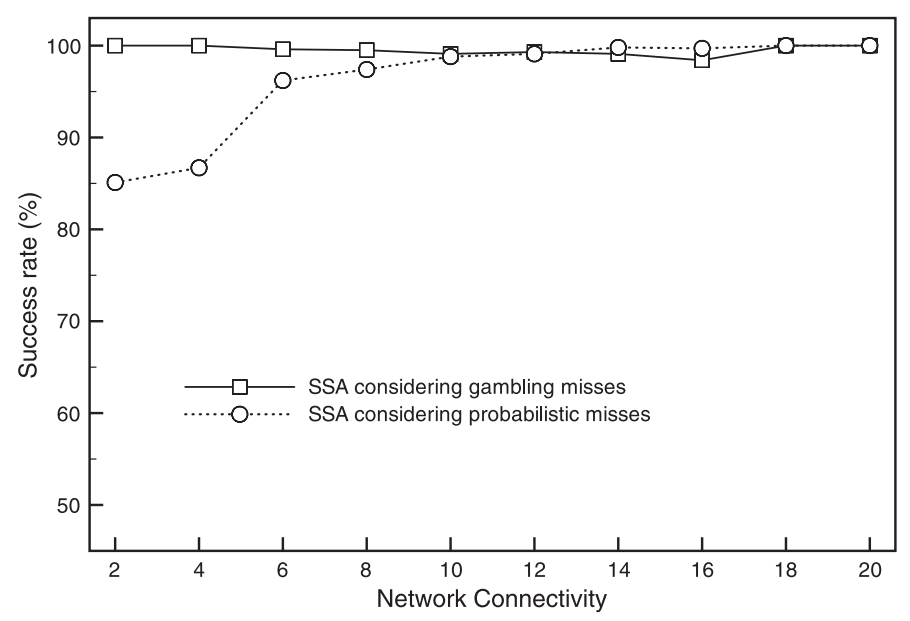

(a)

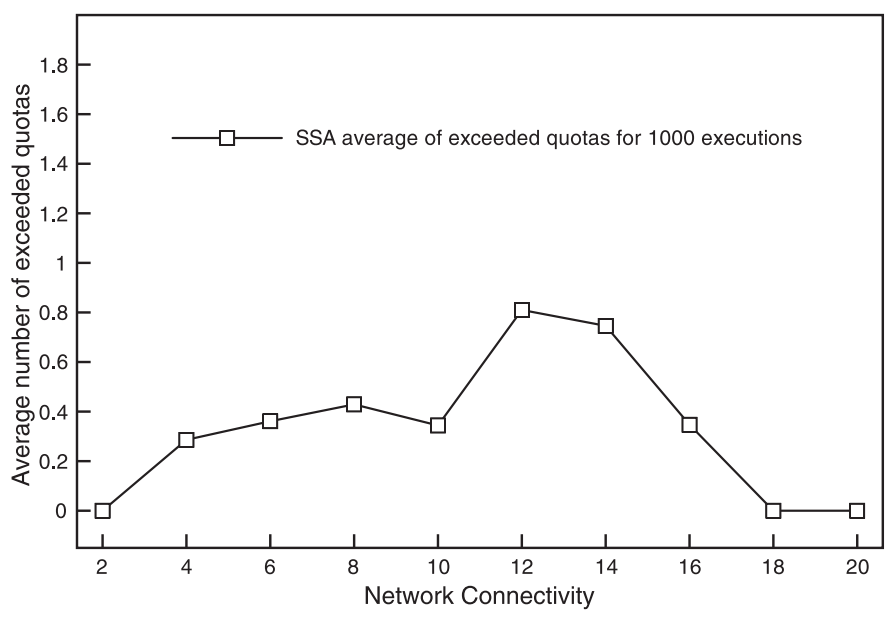

(b)

FIGURE 11. SSA with countermeasure, $L_{i} \in[0.05,0.55]$ and $q_{i}=5$ messages: (a) success rate and (b) average number of exceeded quotas.

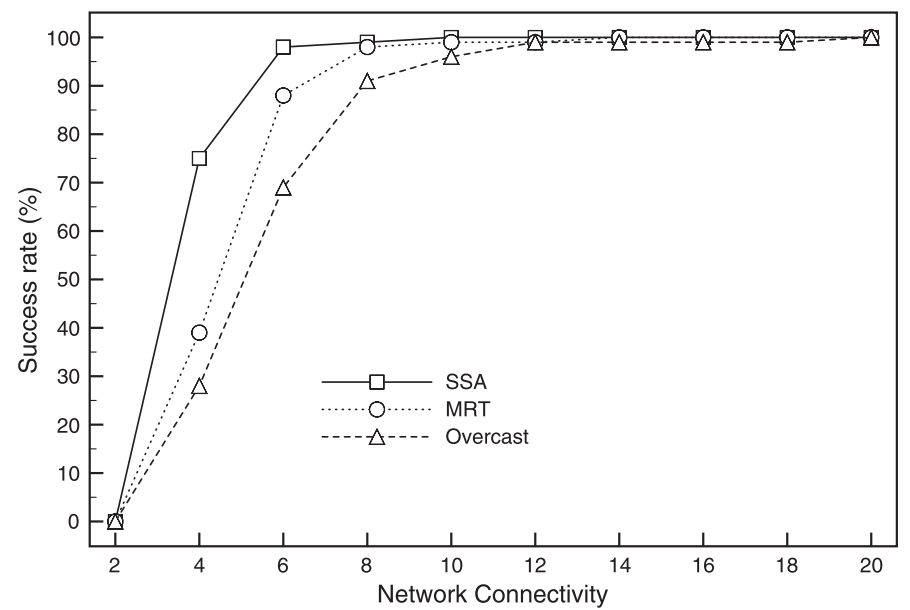

(a)

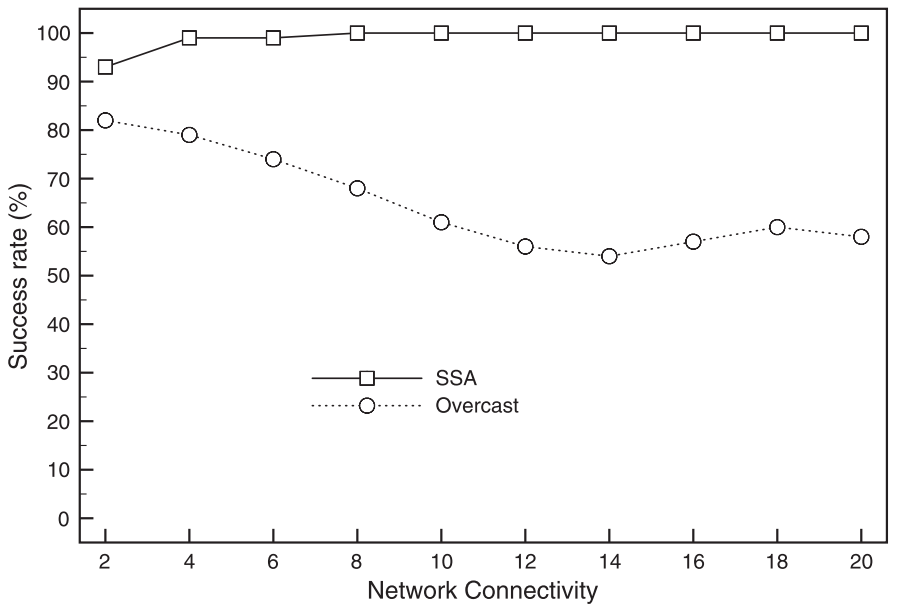

(b)

FIGURE 12. SSA reliability and bandwidth adaptiveness advantage: (a) $q_{i}=c$ and (b) $q_{i}=5$.

behavior than when using the Overcast tree while varying the network connectivity. Indeed, as the connectivity increases more links in the system are created offering a larger choice of links to the MPT-construction technique. While the MPT takes advantage to include more reliable links, the Overcast tree moves away from the line structure that imposes more leaves and thus more lost quotas. These latter quotas would contribute to hide the environment unreliability if not lost.

\subsection{Scalability}

In order to evaluate the scalability of our algorithm, we performed several experiments with our simulation model, by drastically augmenting the number of processes in the system.
In doing so, we considered all links to have the same loss probability $L \simeq 0.05$, and we fixed the scope $d$ to 50 , the incrementing rate $r$ to 40 and the network connectivity $c$ to 4 . We also considered all individual quotas $q_{i}$ to be the same and equal to the network connectivity, i.e. $\forall q_{i}, q_{i}=4$. Our scalability evaluation is pictured in Figs $13 \mathrm{a}$ and $14 \mathrm{a}$, which show the rate of executions that succeeded to reach all nodes (100\% of nodes), $99 \%$ of nodes and $98 \%$ of nodes. In Fig. 13a the number of nodes in the system is varied in a linear way while in Fig. 14a it is varied in an exponential way. Figs $13 \mathrm{~b}$ and $14 \mathrm{~b}$ then show the corresponding countermeasure price, in terms of the average of exceeded quotas needed to handle detectable gambling misses. Based on these figures, we can conclude that our strategy provides a scalable streaming solution, with 


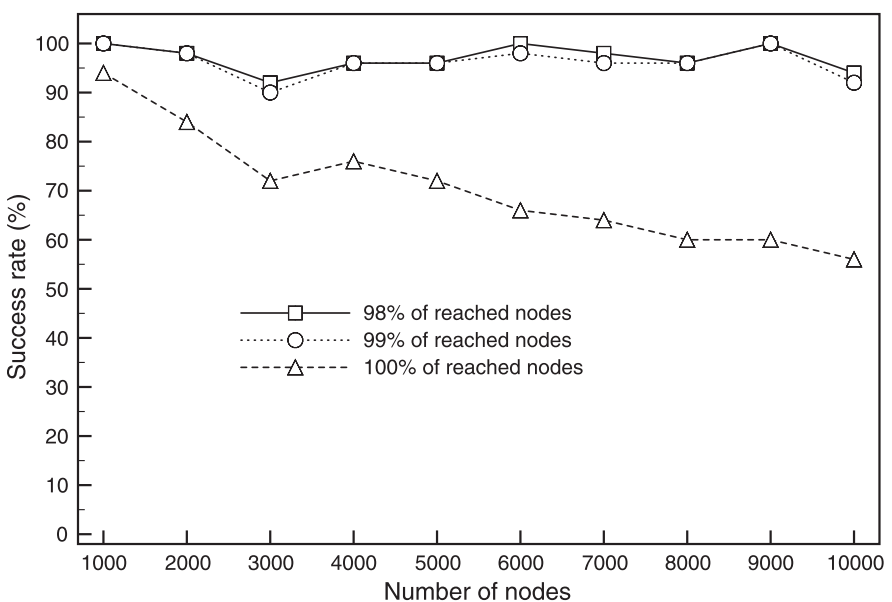

(a)

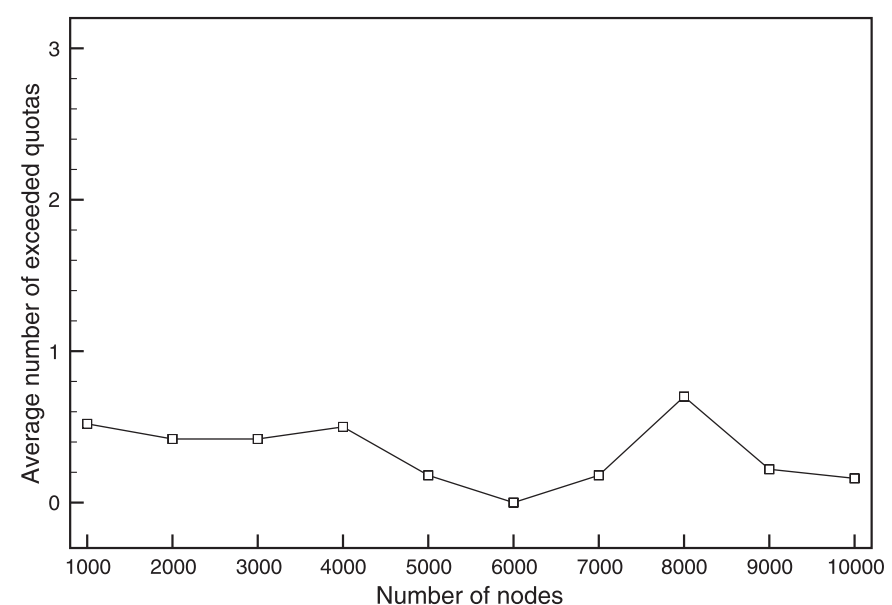

(b)

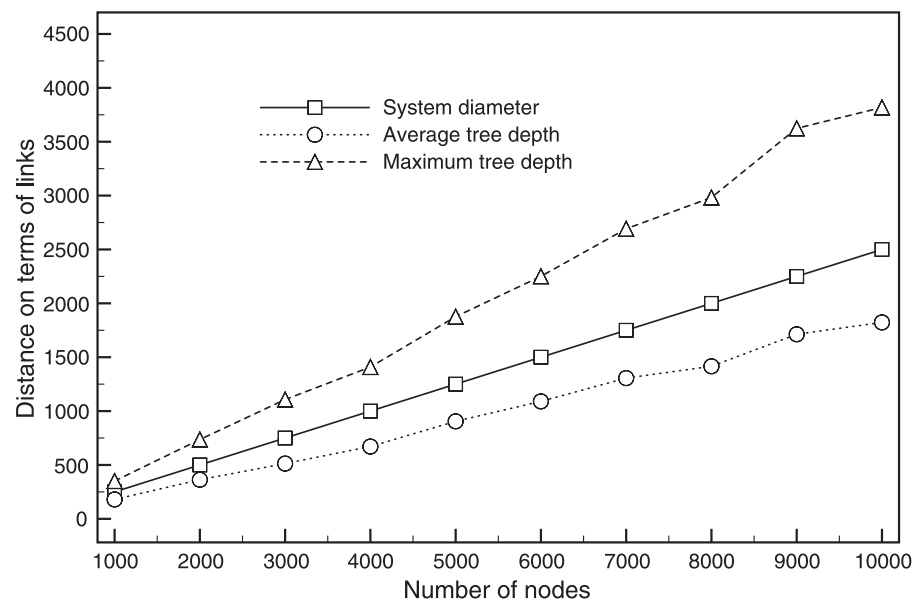

(c)

FIGURE 13. Scalability of SSA with linear growth of nodes, $L \simeq 0.05$ and $q_{i}=4$ messages: (a) success rate, (b) average number of exceeded quotas and (c) system diameter and built tree depth.

a graceful linear decrease as the number of processes in the system increases. We also notice that our solution requires a very small number of exceeded quotas to correct cyclic conflicts.

In each execution we have measured (1) the system diameter, computed as the number of links in the shortest path separating the most distant nodes; (2) the average tree depth, which represents the average distance, in terms of number of links, separating the source node to all the leaves in the resulting propagation graph; and (3) the tree depth in the propagation graph (i.e. maximum distance between the source node and the leaves). These measurements are shown in Figs 13c and 14c. Note that the average tree depth is lower than the system diameter. This shows that while our tree-construction technique aims at using the maximum of available resources, the resulting propagation graph is not a line, although a line is the topology that maximizes the use of quotas. Indeed, when enough quotas are available at some nodes (e.g. at hubs) our MPT-construction algorithm assigns more than one child to those nodes, making the global tree shorter.

\section{RELATED WORK}

Several peer-to-peer streaming solutions have been proposed recently. Mainly, we can classify them into two classes: structured [4-11] and unstructured [12-18]. The unstructured approach usually relies on a gossiping protocol, which consists in having each peer forward the data it receives to a set of randomly chosen neighbors. As a consequence, the path followed by the disseminated data is not deterministic. In contrast, the structured approach consists in first organizing the network peers into some overlay network and in routing disseminated data through this virtual topology.

These two approaches focus on different goals. Initially the structured strategy was devised to adapt to the underlying 


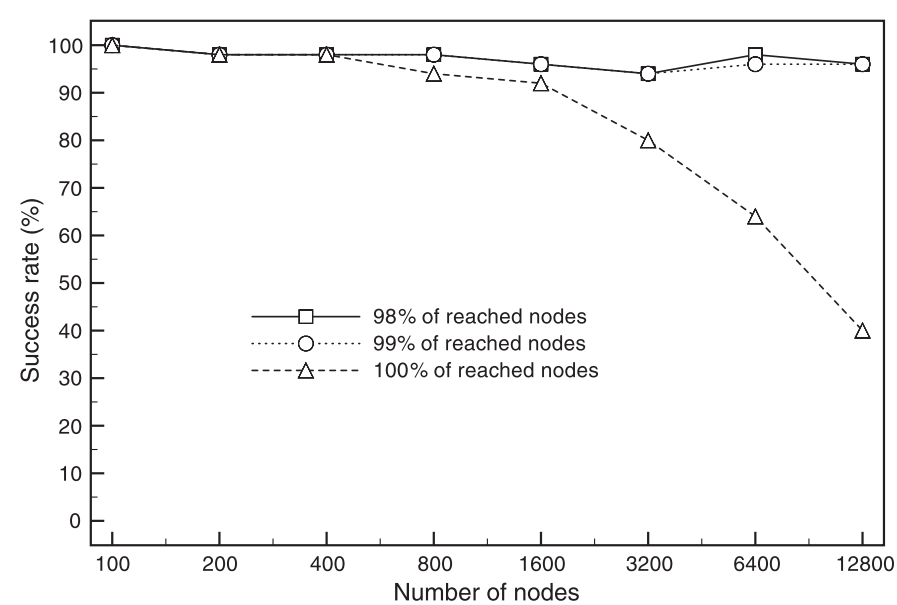

(a)

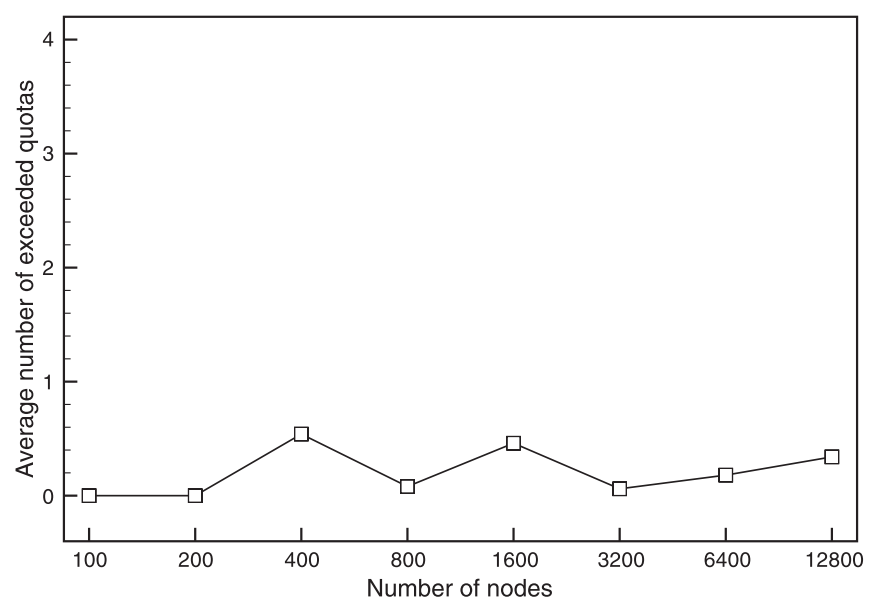

(b)

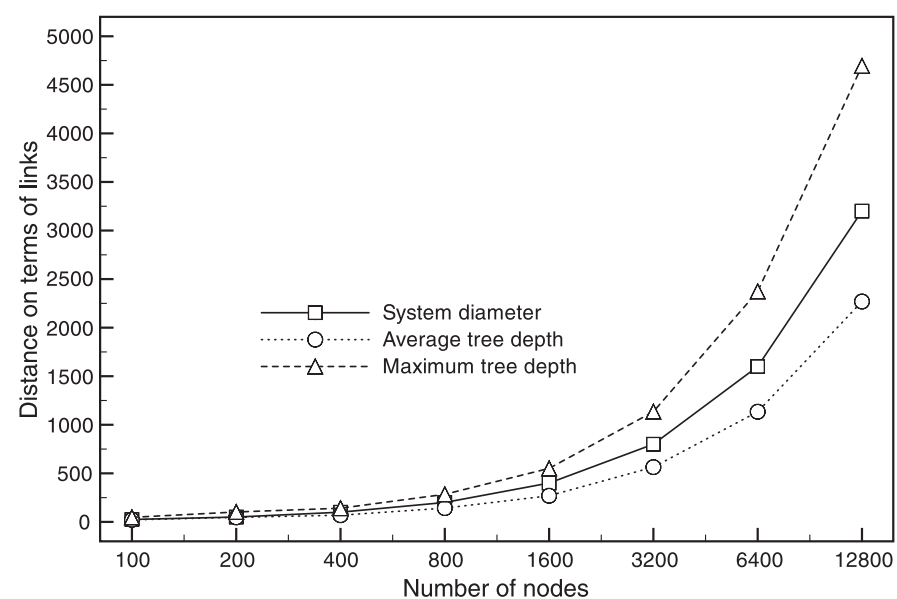

(c)

FIGURE 14. Scalability of SSA with exponential growth of nodes, $L \simeq 0.05$ and $q_{i}=4$ messages: (a) success rate, (b) average number of exceeded quotas and (c) system diameter and built tree depth.

network characteristics, whereas the unstructured strategy, known as network agnostic, was devised for scalability. To ensure the same reliability, a structured dissemination uses fewer messages than an unstructured one. It however assumes that nodes have some knowledge about the network and imposes a computation overhead, which hinders the scalability of these approaches.

Recently several researches worked to reduce the gap between the structured and the unstructured approaches. In the unstructured side, several approaches proposes a more deterministic forward decision in order to adapt some environment constraints or to avoid wasting resources by sending a duplicated message. Along this line, [12, 19, 20] propose gossip-based strategies to ensure either an optimal reliability or an optimal delay by tuning the forward decision based on information about the neighbors received packets. That is, in order to reach some delay or rate targets, each node tries to answer the following question: which stream packets should be forwarded to which neighbor? Similar to our approach, [19] addresses the network links' capacity limitations, however, it does not consider these components' unreliability.

In the structured side, several approaches propose an overlay construction mechanism to approach the scalability of the unstructured strategies. Our solution is part of this category. Along this line, several solutions based on a tree have been proposed in the literature $[4,7,8,21]$. Some of them define a multicast tree that aims at optimizing the bandwidth use $[4,7,22]$. Others also deal with scalability by limiting the knowledge each process has about the system [8, 21]; yet other systems aim at increasing robustness with respect to packet loss [23-25]. Our approach differs from these systems in that it targets the three goals simultaneously. Our propagation structure is built collaboratively by distributed processes using their respective partial views of the system. Reliability is accounted for by each process when building its local tree. Finally, bandwidth constraints are considered when defining how to forward packets along the propagation graph. 
Narada [7] builds an adaptive mesh that includes group members with low degrees and with the shortest path delay between any pair of members. Then, a standard routing protocol is run on the overlay mesh. This work differs from ours by considering latency as the main cost related to links. While using the probing to change links in order to optimize the mesh, Narada does not take into account the loss probability of added or retrieved links. Furthermore, Narada nodes maintain a global knowledge about all group participants. In comparison, we take the process and link failure probabilities into account and maintain local information only.

Regarding the forwarding load distribution, the work closest related to ours is Overcast [4], which leads to deep distribution trees. Such a tree would be our MPT in reliable environments, that is, if links do not lose messages.

Reducing the number of gossip messages exchanged between processes by taking the network topology into account is discussed in $[26,27]$. Processes communicate according to a pre-determined graph with minimal connectivity to attain a desired level of reliability. Similar to our approach, the idea is to define a directed spanning tree on the processes. Different from ours, process and link reliabilities are not taken into account to build such trees.

Our strategy shares some design goals with broadcast protocols such as [1]. Both rely on the definition of criteria for selecting the multicasting graph. In our strategy, however, we strive to both decrease packet loss and balance the forwarding load. The notion of reachability probability of a tree is presented in [1] to define the MRT. In our work, we define the reachability probability of the streaming differently, by considering local knowledge only. These approaches illustrate a trade-off in stream diffusion algorithms: while the protocol in [1] can lead to the optimum propagation tree, it requires global topology knowledge; our current algorithm relies on local knowledge but may not result in the optimal propagation tree.

When it comes to dealing with loops, which naturally appear in decentralized tree-based streaming solutions, several streaming solutions propose tree-computation techniques that consist in dividing multicast members into groups. In such approaches, each group has a leader who is responsible for organizing group members in a subtree, while leaders are in turn also organized in a tree [6,28]. While this strategy prevent loops in the resulting overlay, it however penalizes the efficiency since all optimization are done locally to each group, i.e. nodes in different groups are unable to form overlay links.

Another set of tree-based solutions avoid loop problems by taking advantage of logical-address techniques, traditionally dedicated to routing solutions, in order to build a tree overlay. An example of such solutions is SplitStream [8], which builds several trees based on Scribe [5] and Pastry [29]. This approach ensures scalability since no computation is needed to define the tree. The routing is done implicitly by following the logical addresses assigned to members. The drawback of this approach is the absence of a match between the overlay and the underlying physical network. That is, no efficiency guarantee can be ensured with this approach.

Our approach to detect loops, when building efficient tree overlays, differs from previous ones in that it ensures a resulting global tree close to the one built in a centralized manner, i.e. the tree we would obtain if we had a global knowledge about the system. In [30], we presented an overview of our solution focusing on the tree-building technique, while providing no detail on our loop detection mechanism nor on its handling.

\section{CONCLUSION}

This paper introduces a probabilistic algorithm for reliable stream diffusion in unreliable and constrained environments. Different from more traditional approaches, we resort to a "gambling approach," which deliberately penalizes a few consumers in rare cases, in order to benefit most consumers in common cases. Experimental evaluation has shown that our protocol outperforms GBAs when subject to similar environment constraints. We believe that this may open up new directions for future work on large-scale data dissemination protocols.

\section{FUNDING}

This research is funded by the Swiss National Science Foundation, in the context of Project number 200020-120188.

\section{REFERENCES}

[1] Garbinato, B., Pedone, F. and Schmidt, R. (2004) An Adaptive Algorithm for Efficient Message Diffusion in Unreliable Environments. Proc. DSN'04, Florence, Italy, pp. 507-516. IEEE

[2] Aho, A.V., Hopcroft, J.E. and Ullman, J. (1987) Data Structures and Algorithms, Addison-Wesley.

[3] Tanenbaum, A.S. (2002) Computer Networks. Prentice-Hall PTR, NJ, USA.

[4] Jannotti, J., Gifford, D.K., Johnson, K.L., Kaashoek M.F. and O'Toole, Jr. J.W. (2000) Overcast: Reliable Multicasting with an Overlay Network. Proc. OSDI'00, San Diego, CA, USA, pp. 197-212. USENIX

[5] Castro, M., Druschel, P., Kermarrec, A.M. and Rowstron, A.I.T. (2002) Scribe: a large-scale and decentralized application-level multicast infrastructure. IEEE J. Sel. Areas Commun., 20, 1489-1499.

[6] Banerjee, S., Bhattacharjee, B. and Kommareddy, C. (2000) Scalable Application Layer Multicast. Proc. SIGCOMM'02; New York, NY, USA, pp. 205-217.

[7] Chu, Y., Rao, S. and Zhang, H. (2000) A Case for End System Multicast. Proc. SIGMETRICS'00, Santa Clara, CA, USA, pp. 1-12. ACM

[8] Castro, M., Druschel, P., Kermarrec, A.M., Nandi, A., Rowstron, A. and Singh, A. (2003) SplitStream: High-Bandwidth Multicast in Cooperative Environments. Proc. SOP'03, Bolton Landing, NY, USA, pp. 298-313. ACM 
[9] Francis, P. (2000) Yoid: Extending the Internet Multicast Architecture. AT\&T Center for Internet Research at ICSI (ACIRI).

[10] Mathy, L., Canonico, R. and Hutchison, D. (2001) An Overlay Tree Building Control Protocol. Proc. NGC'01, London, UK, pp. 76-87. Springer.

[11] Tran, D.A., Hua, K.A. and Do, T. (2003) ZIGZAG: An Efficient Peer-to-Peer Scheme for Media Streaming. Proc. INFOCOM'03, San Francisco, CA, USA, pp. 1283-1292. IEEE.

[12] Bonald, T., Massoulié, L., Mathieu, F., Perino, D. and Twigg, A. (2008) Epidemic Live Streaming: Optimal Performance Trade-offs. Proc. SIGMETRICS'08, Annapolis, MD, USA, pp. 325-336. ACM.

[13] Sopcast, http://www.sopcast.com/ (accessed May 1, 2006).

[14] Hei, X., Liang, C., Liang, J., Liu, Y. and Ross, K.W. (2006) Insights into PPLive: A Measurement Study of a Large-Scale P2P IPTV System. Proc. IPTV Workshop'06, Edinburgh, UK.

[15] Zhang, X., Liu, J., Li, B. and Yum, Y.S.P. (2005) CoolStreaming/ DONet: A Data-driven Overlay Network For Peer-to-Peer Live Media Streaming. Proc. INFOCOM'05, Miami, FL, USA, pp. 2102-2111. IEEE

[16] Kermarrec, A.M., Massoulie, L. and Ganesh, A.J. (2001) Probabilistic Reliable Dissemination in Large-scale Systems. Microsoft Research, Cambridge, UK.

[17] Eugster, P., Guerraoui, R., Handurukande, S., Kermarrec, A.-M. and Kouznetsov, P. (2001) Lightweight Probabilistic Broadcast. Proc. DSN'01, Gothenburg, Sweden, pp. 443-452. IEEE.

[18] Coolstreaming, http://www.coolstreaming.us (accessed May 30, 2004).

[19] Massoulié, L., Twigg, A., Gkantsidis, C. and Rodriguez, P. (2007) Randomized Decentralized Broadcasting Algorithms. Proc. INFOCOM'07, Anchorage, AK, USA, pp. 1073-1081. IEEE

[20] Sanghavi, S., Hajek, B. and Massoulié, L. (2007) Gossiping with Multiple Messages. Proc. INFOCOM'07, Anchorage, AK, USA, pp. 2135-2143. IEEE
[21] Kostic, D., Rodriguez, A., Albrecht, J., Bhirud, A. and Vahdat, A. (2003) Using Random Subsets to build Scalable Network Services. USITS'03, Seattle, WA, USA. USENIX.

[22] Zheng, X., Cho, C. and Xia, Y. (2008) Optimal Peer-to-Peer Technique for Massive Content Distribution. Proc. INFOCOM 08, Phoenix, AZ, USA, pp. 151-155. IEEE

[23] Apostolopoulos, J.G. (2001) Reliable Video Communication Over Lossy Packet Networks Using Multiple State Encoding and Path Diversity. Proc. VCIP'01, San Jose, CA, USA, pp. 392-409. SPIE

[24] Apostolopoulos, J.G. and Wee, S.J. (2001) Unbalanced Multiple Description Video Communication Using Path Diversity. Proc. ICIP'01, Thessaloniki, Greece, pp. 966-969. IEEE

[25] Nguyen, T. and Zakhor, A. (2002) Distributed Video Streaming with Forward Error Correction. Int. Packet Video Workshop'02, Pittsburgh PA, USA.

[26] Lin, M.-J. and Marzullo, K. (1999) Directional Gossip: Gossip in a Wide Area Network. University of California, San Diego, CA, USA.

[27] Lin, M.-J., Marzullo, K. and Masini, S. (1990) Gossip Versus Deterministic Flooding: Low Message Overhead and High Reliability for Broadcasting on Small Networks. University of California, San Diego, CA, USA.

[28] Chawathe, Y., McCanne, S. and Brewer E.A. (2000) RMX: Reliable Multicast for Heterogeneous Networks. Proc. INFOCOM'00, Tel Aviv, Israel, pp. 795-804. IEEE

[29] Rowstron, A. and Druschel, P. (2001) Pastry: Scalable, Distributed Object Location and Routing for Large-scale Peerto-Peer Systems. Proc. Middleware'01, Heidelberg, Germany, pp. 329-350. Springer, Berlin.

[30] Allani, M., Garbinato, B., Pedone, F. and Stamenkovic, M. (2007) Scalable and Reliable Stream Diffusion: A Gambling Resource-Aware Approach. Proc. SRDS'07, Beijing, China, pp. 288-300. IEEE. 